\title{
Determinación de glucosa en líquido amniótico como valor predictivo de infección intraamniótica en pacientes con trabajo de parto pretérmino
}

\author{
Rodrigo Baquero MD*, Alejandro Bonivento MD*, Andrés Sarmiento MD*
}

\section{RESUMEN}

OBJETIVO: Determinar la utilidad de la concentración de glucosa en líquido amniótico en pacientes con trabajo de parto pretermino, como factor predictivo de infección intraamniótica.

MATERIAL Y METODOS: Se practicó amniocentesis a 56 gestantes con trabajo de parto pretérmino y membranas íntegras, enviándose muestra para Gram, cultivo y concentración de glucosa. Simultáneamente se obtuvieron muestras de líquido amniótico en 62 pacientes a quienes se les indicó amniocentesis por otras patologías y que sirvieron de grupo de control. Se correlacionaron los resultados con la presencia de parto pretérmino y/o evidencia clínica de corioamnionitis utilizando métodos estadisticos de Chi cuadrado y " $t$ " test.

RESULTADOS: La incidencia de infección intraamniótica determinada como cultivo positivo en pacientes con trabajo de parto pretérmino, fue del $17 \%(10 / 56)$. Un $14 \%$ de pacientes en el grupo problema (8/56) cumplieron criterios clínicos para corioamnionitis. El valor medio de glucosa en pacientes del grupo de control fue de $31.4 \mathrm{mg} / \mathrm{dl}$. El valor medio de glucosa en líquido amniótico en pacientes con corioamnionitis fue de $8.8 \mathrm{mg} / \mathrm{dl}(\mathbf{p}<0.0001)$. Se evidenció correlación de glucosa baja (menor de $14 \mathrm{mg} / \mathrm{dl}$ ) y positividad del cultivo de líquido amniótico con sensibilidad del $87.7 \%$,especificidad del $92.5 \%$, VPP del $46 \%$ y valor predictivo negativo del $99 \%$.

CONCLUSION: La determinación de glucosa en líquido amniótico es una alternativa diagnóstica rápida y certera en la identificación de infección intraamniótica en pacientes con actividad uterina pretérmino.

\section{SUMMARY}

OBJETIVE: To determine the value of amniotic fluid glucose as a predictive test of intraamniotic infection in patients with preterm labor.

METHODS: Amniocentesis was practiced in 56 patients with preterm labor and intact membranes. Determinations of glucose concentration, gram and cultures were practiced in each specimen. Sixty two patients in whom amniocentesis was indicated for other reasons were used as a control group. Results where related to the ocurrence of preterm delivery and/or clinical evidence of chorioamnionitis.

RESULTS: The incidence of intramniotic infection determined as a positive culture, in patients with preterm labor was $17 \%$ (10/56). Fourteen percent $(14 \%)$ of patients in the study group $(8 / 56)$ had clinical diagnostic criteria for chorioamnionitis. The mean glucose concentration in amniotic fluid of patients in the control group was $31.4 \mathrm{mg} / \mathrm{dl}$. Meanwhile the mean glucose concentration in amniotic fluid of patients with chorioamnionitis was $8.8 \mathrm{mg} / \mathrm{dl}(\mathrm{p}<0.0001)$. Low glucose concentrations $(<14 \mathrm{mg} / \mathrm{dl})$ was related to positive cultures in amniotic fluid with a sensitivity of $87.7 \%$, specificity of $92.5 \%$, positive predictive value of $46 \%$ and negative predictive value of $99 \%$.

CONCLUSIONS: The determinations of amniotic fluid glucose concentration is a fast and accurate diagnostic alternative in the identification and management of patients with prreterm labor associated with intramniotic infection.

KEY WORDS: Preterm labor, amniocentesis, chorioamnionitis, glucose.

\section{Introducción}

Con la culminación del estudio actual, que fue realizado durante un período de dos y medios años, se ratifica la importancia que tiene la determinación de glucosa en el líquido amniótico como predictor de infección intraamniótica en pacientes con trabajo de parto pretérmino (1, 9, 20-24).

El esfuerzo del estudio actual tuvo por objeto precisar aún más, el diagnóstico temprano de infección

* Unidad de Medicina Materno-Fetal Sección de Obstetricia y Ginecología, Hospital Militar Central. Santafé de Bogotá. intraamniótica en paciente con trabajo de parto pretérmino debido a la alarmante patología perinatal que de estas pacientes se desprende. Creemos haber cumplido con los objetivos y el propósito enunciado en el actual estudio, así mismo con la confirmación de la hipótesis.

Agradecemos al Hospital Militar Central la colaboración para la realización del mismo, facilitando el uso del Laboratorio de Química y Bacteriología y a las bacteriólogas de dichas secciones para el análisis de las muestras. Hacemos extensivo dicho agradecimiento a los especialistas de la Sección de Ecografía y Alto Riesgo del Servicio de Ginecologia y Obstetricia, y a los residentes rotantes de este servicio que permitieron la recolección de las muestras para este estudio. 


\section{Marco teórico}

Hoy en día el parto pretérmino ocupa un primer lugar como causa de morbilidad perinatal $(1,4,6)$. Se estima que en un $75 \%$ de dicha morbilidad está directamente relacionada a esta patología $(1,4,6)$. A pesar de todos los adelantos tecnológicos con que cuenta la medicina moderna, aún hoy en día no se ha logrado descifrar el parto pretérmino. Su prevención diagnóstico temprano, etiología y manejo siguen siendo objeto de controversia. Aún más, los resultados finales son desalentadores $(2,3,6)$ y muestran como se está fallando en un adecuado enfoque de estas pacientes.

Una de las áreas sobre la cual se ha hecho mayor énfasis en los últimos años es en el área del diagnóstico temprano y etiológico al detectar el inicio de actividad uterina. Existe suficiente evidencia en la literatura obstétrica que relaciona al trabajo de parto pretérmino que no responde a la terapia tocolítica convencional, con la presencia de infección intraamniótica $(2,4,6,8)$. Sin embargo, la demostración de infección intraamniótica con fines útiles terapéuticos, es la mayor parte de las veces inexacta y/o tardía. El Gram de líquido amniótico carece de adecuada sensibilidad (detecta solamente un $50-60 \%$ de pacientes con infección) $(5,9,3,10)$ y el cultivo del mismo, es una prueba más exacta pero que requiere de 3-5 días para su adecuada interpretación (5, 7), tiempo demasiado valioso para el adecuado manejo de estas pacientes. En un gran número de casos el cultivo es técnicamente difícil de llevar a cabo por el tipo de capas comprometidas (5). Otras pruebas más específicas como la cromatografía gas-líquida para el análisis de ácidos orgánicos ha sido propuesta con resultados favorables. Sin embargo, es una prueba que requiere una infraestructura sofisticada, es dispendiosa y a un alto costo, lo que la hace poco práctica.

Tradicionalmente se ha empleado en otras áreas de la medicina la cuantificación de glucosa en fluidos corporales para el diagnóstico de infección. La disminución en la concentración de glucosa en determinado fluido corporal (por ejemplo, líquido cefalorraquídeo) ha sido utilizado como prueba diagnóstica de infección, con un suficiente valor predictivo $(11,13-17)$.

¿Por qué la invasión microbiana de la cavidad amniótica está asociada con una concentración de glucosa reducida? En el LCR, tres mecanismos han sido involucrados para explicar un fenómeno similar.

1. Metabolismo de la glucosa por microorganismos.

2. Consumo de glucosa por neutrófilos activados, y

3. Alternación en el transporte de glucosa a través de la barrera hematoencefálica.

La más aceptada explicación para la baja concentración de glucosa en el líquido cefalorraquídeo, es el metabolismo de la glucosa por las células blancas y bacterias. La sugerencia que el catabolismo de la glucosa por neutrófilos activados es el primer mecanismo que incluye lo siguiente:

1. Neutrófilos obtenidos del líquido cefalorraquídeo séptico consumen glucosa a una rata 2 ó 3 veces mayor que los neutrófilos de pacientes no infectados (15).

2. La incubación de líquido cefalorraquídeo con una combinación de leucocitos y bacterias da como resultado una concentración de glucosa disminuida, mientras que la incubación por uno solo no tiene el mismo efecto (16).

3. Perros irradiados leucopénicos no responden a la meningitis meunocócica con pleocitosis o con reducción de la glucosa en el líquido cefalorraquídeo (17).

En contraste con las infecciones bacterianas, las virales no se asociaron con una reducción en la concentración de glucosa. En las infecciones virales las células mononucleares son predominantes. Es sabido que estas células tienen una rata de consumo de glucosa menor que los neutrófilos.

En los últimos meses han continuado la publicación de nuevos reportes científicos que incitan al uso de la determinación de glucosa en líquido amniótico, como prueba para la detección temprana de infección intraamniótica, como también con valor predictivo para aquellas pacientes que no la han desarrollado y corren riesgo de sufrirla (20-22).

La utilización de ésta, como prueba predictiva de infección intraamniótica, fue propuesta inicialmente hacia finales del 90 y principios del 91 por varios investigadores de Norte América $(1-3,5)$. Se han propuesto valores de concentración de glucosa en líquido amniótico con significancia para predicción de resultados positivos para cultivo de líquido amniótico, que oscilan entre $16 \mathrm{mg} / \mathrm{dl}$ (22) y $14 \mathrm{mg} / \mathrm{dl}$ (5) con una sensibilidad estimada del 79$86 \%$, una especificidad del 91 al $94 \%$, valor predictivo positivo del 63 al $87 \%$, y un valor predictivo negativo del $90-97 \%(5,23)$. Con valores de glucosa menores de $5 \mathrm{mg} /$ dl se ha reportado un valor predictivo positivo del $90 \%$ y con mayores de $20 \mathrm{mg} / \mathrm{dl}$ un valor predictivo negativo del 98\% (23), que comparado con los porcentajes anteriormente nombrados $(5,23)$ son mayores.

Se sugiere también que los niveles de glucosa en líquido amniótico no van a presentar cambios significativos en su concentración, independientemente del tiempo y temperatura de almacenamiento, a no ser que esté o se encuentre presente infección intraamniótica (18).

Esta por dilucidar la sensibilidad y especificidad entre la concentración de glucosa en líquido amniótico y la tinción de Gram en el mismo en el diagnóstico de infección intraamniótica, pero parecer ser que la primera aventaja a la segunda.

En nuestro medio dicha experiencia no se ha llevado a cabo aún. Si tenemos en cuenta el bajo costo de examen, su fácil obtención y los amplios beneficios obtenidos, esta prueba se nos antoja sería ideal en nuestro medio; por lo general limitado económicamente y en recursos técnicos. De demostrarse útil, confirmando la experiencia de los estudios norteamericanos, sería un pilar básico y a bajo costo en el diagnóstico etiológico y temprano de esta patología tan frecuente y de tan alto impacto en el resultado final perinatal.

\section{Problema}

El parto prematuro constituye la primera causa de morbimortalidad perinatal y a pesar de ocupar este lugar, su prevención, diagnóstico temprano, etiología y manejo continúan siendo punto de controversia a tal punto que se detectan fallas en el enfoque de estas pacientes. 


\section{Objetivos}

\section{Objetivos generales}

- Evaluar la utilidad del valor de glucosa en líquido amniótico, como prueba diagnóstica rápida de infección intraamniótica en pacientes con trabajo de parto pretérmino.

\section{Objetivos específicos}

- Relacionar los valores de glucosa con la existencia de infección intraamniótica confirmada por medio de cultivos.

- Determinar el poder predictivo de la glucosa en líquido amniótico como prueba en el diagnóstico de infección intraamniótica.

- Determinar el valor normal de glucosa en líquido amniótico.

- Determinar la frecuencia de infección intraamniótica asociada a trabajo de parto pretérmino.

\section{Propósito}

Establecer un método diagnóstico de corioamnionitis que sea rápido y económico con el fin de facilitar en tiempo y dinero, la detección de esta patología y con esto establecer una conducta pronta en beneficio de la paciente y disminuir costos para la institución con relación a los métodos utilizados habitualmente.

\section{Variables de estudio}

Infección intraamniótica (corioamnionitis)

Valor de glucosa en líquido amniótico.

\section{Hipótesis}

La glucosa en líquido amniótico es menor en pacientes embarazadas con corioamnionitis, con relación a las que no presentan infección.

\section{Materiales y métodos}

Nombre del diseño: Estudio de concordancia de pruebas diagnósticas.

\section{Selección en pacientes}

Criterios de inclusión: Inicialmente pacientes que ingresen al servicio de Ginecobstetricia del HOSMIL, del IMI y Clínica San Rafael con diagnóstico de amenaza de parto prematuro, fueron seleccionadas en el primer período de junio de 1992 a noviembre del mismo año. La ampliación de la muestra se realizó durante un nuevo período de junio de 1993 a noviembre de 1994 con reportes preliminares. El recién nacido producto del embarazo tuvo una evaluación neonatal. La ampliación de la misma muestra de junio de 1993 a noviembre de 1994 se hizo únicamente con pacientes del HOSMIL, por facilidades administrativas, logísticas y de procesamiento standard de las muestras.

Criterios de exclusión: Se excluirán pacientes con diabetes mellitus, ruptura prematura de membranas, pacientes que habían recibido b-miméticos o quienes se negaron a participar en el estudio o no aceptando la amniocentesis.
Técnicas y procedimientos: Las pacientes admitidas al servicio de Ginecobstetricia que reunieron los criterios de inclusión al estudio serán sometidas al siguiente protocolo:

- Confirmación del diagnóstico de trabajo de parto pretérmino (TPP). TPP se define para efectos del estudio como la presencia de actividad uterina regular (por lo menos 2 contracciones en 10 minutos), acompañada de cambios cervicales (dilatación $2 \mathrm{cms}$, borramiento del $80 \%$ ), y/o por lo menos uno de los siguientes: expulsión del tapón, sangrado o sensación de presión pélvica en embarazo menor de 36 semanas.

- Descartar la presencia de amniorrexis por historia clínica, examen físico, prueba de papel de nitrazina y/ o estudio ultrasonográfico.

- Amniocentesis transabdominal aséptica bajo visión ecográfica, con obtención de líquido amniótico para valoración de concentración de glucosa, y cultivo para aerobios y anaerobios.

Las pacientes sin signos clínicos de infección fueron manejadas con tocólisis (terbutalina, sulfato de magnesia o nifedipina) de acuerdo con criterio médico. Su edad gestacional, el resultado del cultivo y su evolución clínica determinaron el posterior manejo. Se consideró como tocólisis fallida la dilatación de por 10 menos $5 \mathrm{cms}$, en presencia de la actividad uterina. El uso de maduración pulmonar estuvo sujeto a la edad gestacional, a la previa toma de la muestra del líquido amniótico y a la sospecha de presencia de infección intraamniótica.

Se definió infección intraamniótica como la presencia de un cultivo positivo de líquido amniótico. Corioamnionitis se definió como la presencia de fiebre $\left(37,5^{\circ} \mathrm{C}\right)$, $\mathrm{y}$ por lo menos dos de los siguientes: taquicardia fetal o materna, sensibilidad uterina, leucocitosis o la presencia de flujo genital fétido. Se consideró sepsis neonatal la presencia de un cultivo positivo en sangre, orina o LCR.

Simultáneamente se practicó determinación de glucosa en líquido amniótico (LA) a un grupo de pacientes sin amenaza de parto prematuro (APP) que sirvieron como grupo control.

La distribución numérica y porcentual de dicho grupo control comparado con el grupo estudio se discrimina en la siguiente tabla. Es de anotar que algunas de las mismas pacientes, presentaron más de una indicación asociada.

\section{Tabla 1}

DISTRIBUCION NUMERICA, PORCENTUAL E INDICACION DE LAS AMNIOCENTESIS

\begin{tabular}{|lcc|}
\hline Indicación & No. Pacientes & Porcentaje \\
\hline APP* & 56 & 47.5 \\
MPF* & 46 & 45.3 \\
Estudio genético & 9 & 7.6 \\
Polihidramnios & 5 & 4.2 \\
Sospecha de isoinmunización & 1 & 0.8 \\
Placenta previa & 1 & 0.8 \\
\hline
\end{tabular}

(*) Amenaza de parto pretérmino

(**) Madurez pulmonar fetal 
Posterior al parto se practicó evaluación neonatal completa buscando detectar infección.

La amniocentesis para tomas de muestra de líquido amniótico fue realizado por los investigadores bajo visión ecográfica directa continua con medidas de asepsia y antisepsia adecuadas, utilizando una aguja calibre 22 . El fluido era aspirado con jeringas estériles, las cuales eran selladas, rotuladas y enviadas de inmediato al laboratorio para estudio bioquímico (glucosa) y bacteriológico (gram y cultivo).

Es de anotar que previo a la realización de la amniocentesis, era determinado el grupo sanguíneo materno y su factor $\mathrm{Rh}$. La importancia de la misma radicó en la determinación de pacientes que se les administró globulina inmune $\mathrm{Rh}$ (Rhesuman) después de la amniocentesis.

El ecógrafo utilizado en el actual estudio es referenciado con la marca Siemens Sonoline SL-2 con transductor sectorial de $3,5 \mathrm{~Hz}$.

La determinación de glucosa en el líquido amniótico fue realizada en el analizador automático de química Hitachi-704, mediante el método GOP-PAP.

\section{Análisis estadístico}

Se practicó análisis estadístico con los métodos de Chi cuadrado y " $\mathrm{t}$ " test.

\section{Aspectos administrativos}

Cada paciente demandó la existencia de una hoja con sus datos respectivos donde se almacenó la información pertinente a la amniocentesis y posteriormente al resultado de la glucosa, el cultivo del líquido amniótico y el resultado neonatal. Dicha hoja fue ajena a la historia clínica y fue de uso exclusivo de los autores del trabajo.

El costo inherente al proyecto estuvo determinado por el costo de la determinación de glucosa, Gram y cultivo en líquido amniótico, el estudio paraclínico neonatal (que se practica de rutina). Los procedimientos de amniocentesis fueron realizados por los autores y el procesamiento de las muestras se hizo efectivo en el laboratorio del HOSMIL.

La Dirección del HOSMIL por intermedio y con la supervisión de la División de Investigación, autorizó el procesamiento de las muestras de líquido amniótico para determinación de glucosa y cultivos para gérmenes comunes por valor de $\$ 800$ per cápita, que fueron subsidiados directamente por la institución.

\section{Criterios éticos}

Toda paciente con amenaza de parto prematuro debe ser estudiada con el fin de descartar infección intraamniótica como factor desencadenante, y esto se logra obteniendo líquido amniótico por punción (amniocentesis).

Este procedimiento forma parte del estudio de estas pacientes, considerándose como necesario y en ningún momento como nuevo instrumento diagnóstico experimental. Por este motivo no se requiere autorización por parte de la paciente excepto la normalmente expresa en la hoja de ingreso a la institución.

\section{Resultados}

Las pacientes seleccionadas e incluidas en el estudio actual, ingresaron en un período comprendido desde el 1o. de junio de 1992 al 30 de noviembre de 1994, con una totalidad de 118 pacientes, distribuidas en 62 pacientes $(52.2 \%)$ del grupo control y 56 pacientes $(47.5 \%)$ casos con diagnóstico de amenaza de parto pretérmino (APP) que cumplieron los criterios de inclusión y exclusión previamente enunciados.

A todas las pacientes incluidas en el estudio se les practicó amniocentesis, gram, cultivo y cuantificación de glucosa en líquido amniótico.

La edad gestacional promedio de la totalidad de las pacientes fue de 30.3 semanas con rangos de 16 a 40 semanas.

El valor medio de glucosa en el líquido amniótico (LA) en el grupo control fue de $31,1 \%$ (62 casos) y de $29.5 \mathrm{mg} \%$ en el grupo estudio (APP).

La edad gestacional promedio en pacientes que cumplieron criterios para corioamnionitis fue de 27 semanas, comparada con la edad gestacional de las pacientes que no tuvieron corioamnionitis que fue de 30.5 semanas.

Se obtuvieron 10 cultivos positivos $(17,8 \%)$ en las pacientes con amenaza de parto pretérmino, sin la necesidad de que las mismas tuvieran clínica de corioamnionitis. Cumplieron requisitos para ser catalogadas en el grupo de corioamnionitis 8 pacientes $(14 \%)$ de las pacientes con APP (grupo de casos).

Dentro del grupo APP el valor medio de glucosa en el LA fue de $29,2 \mathrm{mg} / \mathrm{dl}$, mientras que la misma en el grupo control fue de $32,7 \mathrm{mg} / \mathrm{dl}$ con una $\mathrm{p}=0.371$, que no muestra una diferencia significativa.

Tabla 2

VALOR DE GLUCOSA MEDIA EN LA SEGUN GRUPO DE ESTUDIO

\begin{tabular}{|lcc|}
\hline Grupo & No. Casos & Glucosa Media mg/dl \\
\hline Control & 62 & 32.7 \\
APP & 56 & 29.2 \\
\hline
\end{tabular}

El valor medio de glucosa en LA en pacientes con clínica de corioamnionitis fue de $8.8 \mathrm{mg} / \mathrm{dl}$, mientras que el valor promedio de glucosa en LA en pacientes sin clínica de corioamnio fue de $32,7 \mathrm{mg} / \mathrm{dl}$, con un $\mathrm{p}=0,0001$ que demuestra un valor estadístico significativo.

Tabla 3

VALOR PROMEDIO DE GLUCOSA EN LA EN PACIENTES CON CLINICA DE CORIOAMNIONITIS Y SIN ELLA

\begin{tabular}{|lcc|}
\hline Grupo & No. Pacientes & $\begin{array}{c}\text { Glucosa } \\
\mathrm{mg} / \mathrm{dl}\end{array}$ \\
\hline Corioamnionitis & 8 & 8.8 \\
Sin corioamnionitis & 110 & 32.7 \\
\hline
\end{tabular}

$\mathrm{P}=0.0001$

Al correlacionar la presencia de una glucosa menor de $14 \mathrm{mg} / \mathrm{dl}$ en LA y un cultivo positivo del LA, la sensibilidad de esta prueba fue del $87,7 \%$ y la especifici- 
dad del $92,5 \%$ un valor predictivo positivo del $46 \%$ y un valor predictivo negativo del $99 \%$.

Al correlacionar glucosa menor de $14 \mathrm{mg} / \mathrm{dl}$ con presencia de corioamnionitis, se encontró una relación positiva, con una sensibilidad del $75 \%$, especificidad del $90,9 \%$ y un valor negativo del $98 \%$.

De las pacientes que tuvieron APP, el $19 \%$ tuvieron glucosa baja, con punto de corte menor de $14 \mathrm{mg} / \mathrm{dl}$, que se correlaciona con las referencias sobre la asociación de APP y proceso infeccioso (corioamnionitis).

Al hacer una correlación de las glucosas en el líquido amniótico, excluyendo aquellas pacientes con corioamnionitis tuvieron una tendencia de normalidad con una media de $31.4 \mathrm{mg} / \mathrm{dl}$ con rango entre 28,7 y $34,2 \mathrm{mg} / \mathrm{dl}$.

Las pacientes con glucosa superior al nivel de corte mostraron un control fácil para uteroinhibición de la APP y ninguna presentó cultivo positivo.

\section{Discusión}

la relación de infección meníngea con disminución de la concentración de glucosa en el LCR está claramente definida, y los estudios realizados a nivel mundial hasta la fecha, basados en igual hipótesis en el líquido amniótico, se han dirigido a demostrar dicho resultado.

Con la totalidad de la muestra alcanzada hasta la finalización de este estudio, 62 pacientes control y 56 con amenaza de parto pretérmino (APP), con un total de 118 pacientes, es posible realizar afirmaciones sobre este tema con alguna significancia estadística.
Se demuestra que no hay una diferencia significativa de los valores de glucosa en el grupo control y los casos con amenaza de parto pretérmino y lográndose afirmar que la actividad miometrial no influye sobre los niveles de glucosa intraamniótica.

Se puede determinar de este trabajo que la determinación de glucosa en el líquido amniótico es un medio diagnóstico rápido, de bajo costo y confiable para el diagnóstico de corioamnionitis. Su certeza diagnóstica para infección es similar a la del cultivo de líquido amniótico, y debe considerarse como una alternativa diagnóstica razonable para el diagnóstico de corioamnionitis en pacientes con amenaza de parto pretérmino.

De las pacientes del grupo estudio con APP, 19\% tuvieron niveles de glucosa en el líquido amniótico inferiores al nivel de corte, dato que se correlaciona con las referencias existentes sobre la asociación de APP y proceso infeccioso.

El hallazgo de un cultivo negativo de líquido amniótico en nuestro medio hospitalario, no descarta del todo el estado de corioamnionitis, ya que no se realizan cultivos de rutina para anaerobios y gérmenes tales como el Ureaplasma Urealígticum, que representa un $47 \%$ de los agentes etiológicos de la corioamnionitis, y otros tales como el micoplasma y la clamidia. Lo anterior sustenta aún más la utilidad de la determinación de glucosa en el LA, la cual se puede ver modificada por dichos gérmenes aún con cultivo negativo.

\section{BIBLIOGRAFIA}

1. Dildy G.,Pearlman M., Smith L. Amniotic fluid glucose as a predictor of intramniotic infection in preterm labor and premature rupture of membranes. Am. J. Obstet. Gynecol. 164: 1, SOP meeting San Francisco, USA. Enero 1991.

2. Coultrip L., Norris N., Smith B et al. Usefulness of Amniotic fluid glucose measurement in detection of intramniotic infection. Am. J. Obstet. Gynecol. 164: 1, SOP meeting San Francisco, USA. Enero 1991.

3. Gauthier D., Meyer W., Bienierz A et al. Correlation of amniotic fluid glucose with culture results. Am. J. Obstet. Gynecol. 164: 1, SOP meeting San Francisco, USA. Enero 1991.

4. Romero R., Sirtori M., Oyarzum E et al. Infection and labor. Am. J. Obstet. Gynecol. 1989; 161: 817.

5. Romero R., Jiménez C., Lohda et al. Amniotic fluid concentration: A rapid and simple method for the detection of intramniotic infection in preterm labor. Am. J. Obstet. Gynecol. 1990; 163: 968.

6. Morrison J. Preterm labor: A puzzle worth solving. Obstet. Gynecol. 1990; 76: 55.

7. Gibbs R., Blanco J., St Clair O et al. Quantitative bacteriology of amniotic fluid from woman with clinical intramniotic infection at term. J. Inf. Disease 1982; 145: 1.

8. I. Romero R., Mazor M. Infection and preterm labor. Clin. Obstet. Gynecol. 1988; 31: 553-584.

9. Romero R., Emamian M., Quintero R et al. The value and limitations of the Gram stain examination in the diagnosis of intramniotic infection. Am. J. Obstet. Gynecol. 1988; 159: 114-119.

10. Romero R., Emamian M., Quintero R et al. Diagnosis of intraamniotic infectin: the acridine orange stain. Am. J. Perinatol 1989; 6: 41-45.

11. Overtturf GD. Infections of the centralnervous sytems IN: Hoeprich PD. Jordan MC, eds. Infectious diseases. 4th ed. Philadelphia; JB Lippincott, 1989; 114-1132.

12. Brody JS. Diseases of the pleura, mediastinum, diaphragm and chest wall. In: Wyngaarden JB, Smith LH, Jr. eds. Cecil textbook of medicine. 17th ed. Philadelphia; WB Saunders, 1985; 447-454.
13. Parker RH. Skeletal infections. In: Hoeprich PD, Jordan Mc. eds. Infectious diseases. 4th ed. Philadelphia; JB Lippincott, 1989; 13761382.

14. Brooke RD. Alterations in the glucose transport mechanism in patients with complications of bacterial meningitis. Pediatric 1964; 34: 491 501.

15. Betz G., Mauer AM. Glucose consumptiom by polymorphonuclear leukocytes in the cerebrospinal fluid of patients with bacterial meningitis. J. Pediatr. 1967; 70: 767-771.

16. Peersdorf RG., Swarner DR., García M. Synergistic action of pneumococci and leukocytes in lowering cerebrospinal fluid glucose. Proc. Soc. Exp. Biol. Med. 1960; 103: 380-382.

17. Petersdorf RG., García M., Swarner DR. Mechanism of hypoglycorrhachia in experimental peneumococcal meningitis. Proc. Soc. Exp. Biol. Med. 1959; 102: 669-672.

18. Meyer WJ., Gauthier DW. Effect of time and storage temperature on amniotic fluid glucose concentration. Obstet. Gynecol. 1992; 80(6): 1017-1019.

19. Coultrip LL., Grossman JH. Evaluation of rapid diagnostic tests in the detection of microbial invasion of the amniotic cavity. Am. J. Obstet. Gynecol. 1992; 167(5): 1231-1242.

20. Gauthier DW., Meyer JW. Comparison of gram stain, leukocyte esterase activity, and amniotic fluid glucose concentration in predicting amniotic fluid culture results in preterm premature rupture of membranes. Am. J. Obstet. Gynecol. 1992; 167(4): 1092-1095.

21. Gonen R. Amniotic fluid glucose and intraamniotic infection: sensitivity, specificity, and predictive values [letter] Am. J. Obstet. Gynecol. 1992; 166(6): 1863-1864.

22. Gauthier DW., Meyer WJ. Correlation of amniotic fluid glucose concentration and intraamniotic infection in patients with preterm labor or premature rupture of membranes. Am. J. Obstet. Gynecol. 1991; 165(4): 1105-1110.

23. Kiltz RJ., Burke MS. Amniotic fluid glucose concentration as a marker for intraamniotic infection. Obstet. Gynecol. 1991; 78(4): 619-622.

24. Kirshon B., Rosenfeld B. Amniotic fluid glucose and intraamniotic infection. Am. J. Obstet. Gynecol. 1991; 164(3): 818-820. 